\title{
Brassinosteroid Ameliorates Zinc Oxide Nanoparticles-Induced Oxidative Stress by Improving Antioxidant Potential and Redox Homeostasis in Tomato Seedling
}

\author{
Mengqi Li ${ }^{1 \dagger}$, Golam J. Ahammed ${ }^{1 *}$, Caixia $\mathrm{Li}^{1}$, Xiao Bao ${ }^{1}$, Jingquan $\mathrm{Yu}^{1,2,3}$, \\ Chunlei Huang ${ }^{4,5,6 *}$, Hanqin Yin ${ }^{4,5,6}$ and Jie Zhou ${ }^{1,2 *}$
}

OPEN ACCESS

Edited by:

Naser A. Anjum,

University of Aveiro, Portugal

Reviewed by:

Elisa Pellegrini,

Department of Agriculture, Food and Environment, Italy

Xin Li,

Tea Research Institute, Chinese Academy of Agricultural Sciences,

China

*Correspondence:

Jie Zhou

jie@zju.edu.cn;

Golam J. Ahammed

jalal@zju.edu.cn;

Chunlei Huang

583048412@qq.com

${ }^{\dagger}$ These authors have contributed equally to this work.

Specialty section: This article was submitted to Plant Physiology,

a section of the journal Frontiers in Plant Science

Received: 25 March 2016 Accepted: 21 April 2016

Published: 09 May 2016

Citation:

LiM, Ahammed GJ, LiC, BaoX, Yu J, Huang C, Yin H and ZhouJ (2016) Brassinosteroid Ameliorates Zinc Oxide Nanoparticles-Induced

Oxidative Stress by Improving Antioxidant Potential and Redox Homeostasis in Tomato Seedling.

Front. Plant Sci. 7:615.

doi: 10.3389/fpls.2016.00615
' Department of Horticulture, Zijingang Campus, Zhejiang University, Hangzhou, China, ${ }^{2}$ Zhejiang Provincial Key Laboratory of Horticultural Plant Integrative Biology, Hangzhou, China, ${ }^{3}$ Key Laboratory of Horticultural Plants Growth, Development and Quality Improvement, Agricultural Ministry of China, Hangzhou, China, ${ }^{4}$ School of Environmental Studies, China University of Geosciences, Wuhan, China, ${ }^{5}$ Geological Research Center for Agricultural Applications, China Geological Survey, Hangzhou, China, ${ }^{6}$ Zhejiang Institute of Geological Survey, Hangzhou, China

In the last few decades use of metal-based nanoparticles (MNPs) has been increased significantly that eventually contaminating agricultural land and limiting crop production worldwide. Moreover, contamination of food chain with MNPs has appeared as a matter of public concern due to risk of potential health hazard. Brassinosteroid has been shown to play a critical role in alleviating heavy metal stress; however, its function in relieving zinc oxide nanoparticles (ZnO NPs)-induced phytotoxicity remains unknown. In this study, we investigated the potential role of 24-epibrassinolide (BR) in mitigating ZnO NPs-induced toxicity in tomato seedlings. Seedling growth, biomass production, and root activity gradually decreased, but Zn accumulation increased with increasing ZnO NPs concentration (10-100 mg/L) in growth media ( $1 / 2 \mathrm{MS})$. The augmentation of $\mathrm{BR}(5 \mathrm{nM})$ in media significantly ameliorated $50 \mathrm{mg} / \mathrm{L}$ ZnO NPs-induced growth inhibition. Visualization of hydrogen peroxide $\left(\mathrm{H}_{2} \mathrm{O}_{2}\right)$, and quantification of $\mathrm{H}_{2} \mathrm{O}_{2}$ and malondialdehyde (MDA) in tomato roots confirmed that $\mathrm{ZnO}$ NPs induced an oxidative stress. However, combined treatment with $\mathrm{BR}$ and $\mathrm{ZnO} \mathrm{NPs}$ remarkably reduced concentration of $\mathrm{H}_{2} \mathrm{O}_{2}$ and MDA as compared with $\mathrm{ZnO} N P$ s only treatment, indicating that BR supplementation substantially reduced oxidative stress. Furthermore, the activities of key antioxidant enzymes such as superoxide dismutase (SOD), catalase, ascorbate peroxidase and glutathione reductase were increased by combined treatment of $\mathrm{BR}$ and $\mathrm{ZnO} N P s$ compared with ZnO NPs only treatment. BR also increased reduced glutathione (GSH), but decreased oxidized glutathione (GSSG)] and thus improved cellular redox homeostasis by increasing GSH:GSSG ratio. The changes in relative transcript abundance of corresponding antioxidant genes such as CU/Zn SOD, CAT1, GSH1, and GR1 were in accordance with the changes in those antioxidants under different treatments. More importantly, combined application of BR and ZnO NPs significantly decreased $\mathrm{Zn}$ content in both shoot and root of tomato seedlings as compared with 
ZnO NPs alone. Taken together, this study, for the first time, showed that BR could not only improve plant tolerance to ZnO NPs but also reduce the excess zinc content in tomato seedlings. Such a finding may have potential implication in safe vegetable production in the MNPs-polluted areas.

Keywords: brassinosteroids, food safety, hydrogen peroxide, nanotoxicity, oxidative stress, tomato, zinc oxide nanoparticles

\section{INTRODUCTION}

Nanoparticles (NPs) are particles that have at least one dimension less than $100 \mathrm{~nm}$; but have a greater surface area compared to bulk products. In the recent years, engineered NPs are extensively being used for manufacturing variety of industrial, commercial, and medical products that are eventually released to the environment. Thus extensive use of NPs has become a matter of public concern due to potential contamination of food chain by metal-based NPs (MNPs). Zinc oxide NPs (ZnO NPs) are one of the MNPs that are commonly investigated with regards to human and ecosystem health as well as nanotoxicological effect on plants (Chen et al., 2015; Van Aken, 2015; Zhang et al., 2015). Given that $\mathrm{ZnO}$ NPs are wide band-gap semiconductors that exhibit near UV emission and transparent conductivity, their application as electronic sensors and solar voltaic has become common (Lin and Xing, 2008; Ma et al., 2013). Moreover, ZnO NPs are widely exploited for their photolytic properties and are also extensively used in personal care products for their ultraviolet-blocking ability (Hoffmann et al., 1995; Ali et al., 2008). Earlier studies have already reported the presence of $\mathrm{ZnO}$ NPs in sewage treatment plant effluents and in sludge-treated soils used for agriculture (Ma et al., 2013). Another potential formulation of ZnO NPs for agricultural applications could be as a pesticide because of their antimicrobial properties (He et al., 2011; Dimkpa et al., 2013).

Earlier studies on nanotoxicity in plants showed differential effects of MNPs that include positive, negative or no effects on plants (Dietz and Herth, 2011). Nonetheless, most of those studies addressed easily observable parameters, such as germination rate and growth-related features. Plant responses to MNPs not only depend on dose, but also on the species of plants (Chichiriccò and Poma, 2015). For instance, ZnO NPs caused a dose-dependent inhibition in seed germination of cabbage (Brassica oleracea var. capitata L.), while it showed no negative effects on germination of maize (Zea mays L.) seeds (Pokhrel and Dubey, 2013). Moreover, ZnO NPs (1,000 mg/L) reduced root length of corn and cucumber (Cucumis sativus L.), but exhibited no effects on their seed germination (Zhang et al., 2015). In comparison with $\mathrm{Zn}^{2+}$, toxicity of $\mathrm{ZnO}$ NPs on the root elongation of corn could be attributed to the nanoparticulate $\mathrm{ZnO}$, while released $\mathrm{Zn}$ ion from $\mathrm{ZnO}$ could solely contribute to the inhibition of root elongation in cucumber (Zhang et al., 2015).

On the basis of NP size, shapes, compositions and atomic arrangement, interaction of NPs with cellular structure varies a lot and yet very complex and poorly understood (Van Aken, 2015). Moreover, studies on the mechanism of interaction between NPs such as ZnO NPs and plant cell biomolecules are scanty. A few studies on NPs ecotoxicity suggested several potential mechanisms through which $\mathrm{ZnO}$ NPs cause damage to plants (Chen et al., 2015; Zhang et al., 2015). MNPs enter plant tissues, predominantly through pores in the cell wall and endocytosis pathway, and then are translocated through vascular system (Chichiriccò and Poma, 2015). First, the release of $\mathrm{Zn}^{2+}$ from ZnO NPs possibly causes phytotoxicity (Lin and Xing, 2008). Second, ZnO NPs due to their small sizes and large surface area may directly interact with biomolecules and disrupt membranes or DNA (Ma et al., 2013). Most importantly, ZnO NPs promote the generation of reactive oxygen species (ROS) such as superoxide radical $\left(\mathrm{O}_{2}^{\bullet-}\right)$ and hydrogen peroxide $\left(\mathrm{H}_{2} \mathrm{O}_{2}\right)$ in the absence of photochemical energy (Hernandez-Viezcas et al., 2011). It is suggested that excessive generation of ROS can induce membrane lipid peroxidation and cellular damage, which is considered as one of the primary reasons contributing to nanotoxicity in plants (Chichiriccò and Poma, 2015). Plants possess a well equipped antioxidant systems composed of both enzymatic and non-enzymatic antioxidants to scavenge excessive level of ROS that are injurious to cells (Baxter et al., 2014). Exposure of plants to NPs also stimulates antioxidant system in plants, perhaps as an adaptive response to alleviate oxidative stress.

In general, $\mathrm{Zn}$ is required for various metabolic processes in human. However, daily intake that exceeds recommended dietary allowance may cause Zn toxicity (Fosmire, 1990). Ingestion of excess $\mathrm{Zn}$ causes symptoms like nausea, vomiting, epigastric pain, abdominal cramps, and diarrhea in human. Furthermore, the use of $\mathrm{Zn}$ supplements can interfere with the utilization of copper, impair immune function and severely affect lipoprotein profiles (Fosmire, 1990). Vegetables are an integral part of human diet that serves as a major route for intake of essential and/or non-essential metals. An earlier study conducted in a metal-polluted area (Zinc Plant in Huludao City, China) showed high transfer factor values of $\mathrm{Cd}, \mathrm{Zn}$, and $\mathrm{Cu}$ from soil to vegetable (Zheng et al., 2007). Authors of that study calculated total metal target hazard quotients due to consumption of vegetables, which indicated highest health risks to inhabitants close to Zinc plant. Thus, extensive utilization of $\mathrm{ZnO} \mathrm{NP}$ and deliberate release to environment may increase possible health risks to population of metal-polluted areas through the food chain transfer. Therefore, elucidation of strategies that improve plant tolerance to MNP as well as reduce metal uptake are urgent to ensure food security and food safety. Till date, studies relating to alleviation of ZnO NPs-induced phytotoxicity are scanty. Recently, Chen et al. (2015) reported that nitric oxide (NO), a ubiquitous signal molecule in plants could ameliorate $\mathrm{ZnO}$ NPs-induced phytotoxicity in rice seedlings. A number of earlier studies 
relating to enhancement strategies of plant tolerance against heavy metal stress (not MNPs) revealed that signal molecule including phytohormones could alleviate heavy metal-induced phytotoxicity in a range of plant species (Ahammed et al., 2014). For instance, (Ramakrishna and Rao, 2012, 2015) reported that 24-epibrassinolide (a steroidal phytohormone) could alleviate $\mathrm{Zn}^{2+}$ stress by improving antioxidant potential and redox state in radish (Raphanus sativus L.) seedlings. $\mathrm{ZnO}$ NPs can also induce $\mathrm{Zn}^{2+}$ stress by releasing $\mathrm{Zn}$ ions. Thus it is quite likely to anticipate a protective role of brassinosteroids (BRs) against MNPs too.

Brassinosteroids are a class of plant-specific essential steroidal hormones that regulate broad aspects of plant growth, development and responses to various biotic and abiotic stresses (Cui et al., 2011; Ahammed et al., 2014; Zhou et al., 2014; Divi et al., 2016). However, the mechanisms that control BRs-induced stress tolerances are largely unknown (Divi et al., 2016). We previously showed that BRs-induced oxidative stress tolerance involves transient accumulation of hydrogen peroxide $\left(\mathrm{H}_{2} \mathrm{O}_{2}\right)$ that activates antioxidant system in cucumber and tomato plants (Cui et al., 2011; Nie et al., 2013; Zhou et al., 2014). Although BRs initially induce NADPH-based $\mathrm{H}_{2} \mathrm{O}_{2}$ production, it eventually enhances ROS scavenging by stimulating antioxidative machinery, indicating a dual role of $\mathrm{H}_{2} \mathrm{O}_{2}$ in mediating BR-induced stress tolerance (Ahammed et al., 2014). We also showed that BRs could efficiently ameliorate cadmium (a major toxic heavy metal)-induced oxidative stress and photosynthetic inhibition in tomato plants (Ahammed et al., 2013). However, the effect of BRs on MNPs-induced phytotoxicity still remains elusive. In the current study, we investigated the potential role of 24-epibrassinolide (BR, a bioactive BRs) in mitigating $\mathrm{ZnO}$ NPs-induced toxicity in tomato seedlings. This study is expected to provide a better insight into the role of BRs in MNPs-induced oxidative stress that may be useful to ensure food safety in MNPs-polluted areas.

\section{MATERIALS AND METHODS}

\section{MNPs Preparation}

The $\mathrm{ZnO}$ NPs (diameters varying between 20 and $30 \mathrm{~nm}$, a purity $>99 \%$ ) were purchased from the Aladdin Corporation (Shanghai, China). Culture dispersion of NPs was achieved by adding Phytagel (Sigma-Aldrich, St. Louis, MO, USA) powder and a suitable amount of NPs to ultrapure water, and the dispersions were sufficiently shaken after sonication to break up agglomerates. Each concentration of NPs treatment was prepared separately, without dilution, by weighing particles and dispersing them in solid half strength Murashige and Skoog ( $1 / 2 \mathrm{MS})$ medium. The addition of NPs ranged from 10 to $100 \mathrm{mg} / \mathrm{L}$. It is noteworthy to mention that the agar culture medium has the advantage of easy dispersion of NPs without precipitation.

\section{Plant Materials and Treatments}

Authentic tomato seeds (Solanum lycopersicum L. cv. Hezuo903)were sterilized in a $10 \%$ sodium hypochlorite solution for $15 \mathrm{~min}$, rinsed thoroughly with deionized water several times, and subsequently placed in sterilized solid $1 / 2$ MS medium at a controlled temperature of $28^{\circ} \mathrm{C}$ in the dark. After $48 \mathrm{~h}$, the seeds were checked for the germination, and seeds that had sprouted were used in the test. The toxicity tests were conducted in a tissue culture bottle $(240 \mathrm{~mL})$. Each test unit contained $40 \mathrm{~mL}$ of $1 / 2 \mathrm{MS}$ culture media with a specific concentration of $\mathrm{ZnO}$ NPs. Ten tomato seedlings were placed just above the surface of the medium of the test units. The test units were placed in a sterile room. After an incubation period of 15 days, the plants were separated from the agar media, and seedling growth was measured. The NP concentrations of 0,10 , 20, 50, and $100 \mathrm{mg} / \mathrm{L}$ were prepared in four replicate test units per treatment.

In the BR treatment, the $5 \mathrm{nM}$ 24-epibrassinolide was added in the $1 / 2$ MS medium in the process of the solidification. The concentration of BR was chosen based on preliminary dose trial (data not shown).

\section{Measurement of Morphological Parameters and Root Activity}

Morphological parameters such as fresh weight and length of shoot and root, and root activity were determined following exposure of tomato seedlings to different levels of $\mathrm{ZnO}$ NPs for 15 days. In each replicate, 10 plants were selected randomly, and length and fresh weight of each plant were determined. Average values of these 10 plants were considered as one replicate. For determination of root activity, three replicates for each ten treatments such as CK, 10, 20, 50, and $100 \mathrm{mg} / \mathrm{L}$ $\mathrm{ZnO}$ NPs were selected. Roots were washed thoroughly with distilled water and finally with deionized water and cut into small pieces of 3-4 mm. A $0.5 \mathrm{~g}$ portion of these roots sample was placed into tube; $5 \mathrm{~mL} 0.4 \%$ TTC (triphenyl tetrazolium chloride) and $5 \mathrm{~mL} 0.1 \mathrm{mM}$ phosphatic buffer solution ( $\mathrm{pH} 7.0$ ) were added to the tube and allowed to react for $2 \mathrm{~h}$ at $37^{\circ} \mathrm{C}$. Afterward, $2 \mathrm{~mL}$ of $1 \mathrm{M} \mathrm{H}_{2} \mathrm{SO}_{4}$ was added to the tube to stop the reaction. The root activity was expressed by the amount of TPF (triphenyl formazan) deoxidized by TTC (Islam et al., 2007).

\section{Determination of $\mathrm{H}_{2} \mathrm{O}_{2}$ Contents and Histochemical Detection of $\mathrm{H}_{\mathbf{2}} \mathrm{O}_{\mathbf{2}}$}

To determine the $\mathrm{H}_{2} \mathrm{O}_{2}$ concentration, $0.3 \mathrm{~g}$ of fresh root tissues was homogenized in $3 \mathrm{~mL}$ of precooled $\mathrm{HClO}_{4}(1.0 \mathrm{M})$ using a pre-chilled mortar and pestle, according to the method of Willekens et al. (1997).

$\mathrm{H}_{2} \mathrm{O}_{2}$ production in root tissues was monitored using 2,7dichlorofluorescein diacetate $\left(\mathrm{H}_{2} \mathrm{DCF}-\mathrm{DA}\right)$ as described by $\mathrm{Yi}$ et al. (2015). Detached roots were washed with deionized water and incubated $15 \mathrm{~min}$ with $25 \mu \mathrm{M} \mathrm{H} \mathrm{H}_{2}$ DCF-DA in $200 \mathrm{mM}$ phosphate buffer $(\mathrm{pH}$ 7.4) and then washed five times with the same buffer without the dye. To scavenge $\mathrm{H}_{2} \mathrm{O}_{2}$, the root segments were incubated with $1 \mathrm{mM}$ ascorbate or $100 \mathrm{U} / \mathrm{mL}$ catalase for $30 \mathrm{~min}$ that served as negative controls. Fluorescence was observed using a Leica DM4000B microscope and images were captured using a Leica DFC425C camera and the Leica 
application suite V3.8 software (Leica Microsystems, Wetzlar, Hessen, Germany).

\section{Determination of Lipid Peroxidation}

To determine level of lipid peroxidation in roots, concentration of malondialdehyde (MDA) was measured by the 2thiobarbituricacid (TBA) test. Root samples $(0.5 \mathrm{~g})$ were homogenized in $5 \mathrm{~mL}$ of $10 \%(\mathrm{w} / \mathrm{v})$ trichloroaceticacid (TCA). The homogenates were centrifuged at $3,000 \mathrm{~g}$ for $10 \mathrm{~min}$ and $4 \mathrm{~mL}$ of $20 \%$ TCA containing $0.65 \%(\mathrm{w} / \mathrm{v}$ ) TBA was added to $1 \mathrm{~mL}$ of the supernatant. The mixtures were heated in a hot water bath at $95^{\circ} \mathrm{C}$ for $25 \mathrm{~min}$ and immediately placed in an ice bath to stop the reaction. After centrifugation at $3,000 \mathrm{~g}$ for $10 \mathrm{~min}$ and the absorbance of the supernatants was recorded at 440,532 , and $600 \mathrm{~nm}$. The MDA equivalents were calculated according to Hodges et al. (1999).

\section{Determination of Antioxidant Enzyme Activity}

Antioxidant enzymes were extracted by grinding the root tissue $(0.3 \mathrm{~g})$ with $3 \mathrm{~mL}$ ice-cold $50 \mathrm{mM}$ phosphate buffer $(\mathrm{pH} 7.8)$ containing $0.2 \mathrm{mM}$ EDTA and $2 \%$ polyvinylpyrrolidone (w/v). The homogenates were centrifuged at $4^{\circ} \mathrm{C}$ for $20 \mathrm{~min}$ at $12,000 \mathrm{~g}$, and the resulting supernatants were used for the determination of enzymatic activity.

Superoxide dismutase (SOD) activity was assayed by inhibiting the photochemical reduction of nitro blue tetrazolium (NBT) according to Stewart and Bewley (1980). The absorbance was monitored in $560 \mathrm{~nm}$. One unit of SOD is the amount of extract that gives $50 \%$ inhibition of the reduction rate of NBT. Catalase (CAT) activities were determined following the methods of Cakmak and Marschner (1992). The reaction mixture for CAT consisted of $25 \mathrm{mM}$ phosphate buffer ( $\mathrm{PH}$ 7.0), $10 \mathrm{mM} \mathrm{H}_{2} \mathrm{O}_{2}$ and enzyme extract. The decomposition of $\mathrm{H}_{2} \mathrm{O}_{2}$ determined at $240 \mathrm{~nm}$ for $20 \mathrm{~s}$ $\left(E_{240}=39.4 \mathrm{mM}^{-1} \mathrm{~cm}^{-1}\right)$. Ascorbate peroxidase (APX) was measured in a reaction mixture containing $25 \mathrm{mM}$ phosphate buffer (PH 7.0), $5 \mathrm{mM}$ ascorbic acid (ASA), $20 \mathrm{mM} \mathrm{H} \mathrm{H}_{2} \mathrm{O}_{2}$ and enzyme extract with the principle of monitoring the rate of ascorbate oxidation at $290 \mathrm{~nm}$ for $20 \mathrm{~s}$ $\left(E_{290}=2.8 \mathrm{mM}^{-1} \mathrm{~cm}^{-1}\right)$, according to Nakano and Asada (1981). Glutathione reductase (GR) activity was determined according to Foyer and Halliwell (1976). The reaction mixture for GR contained $25 \mathrm{mM}$ phosphate buffer ( $\mathrm{PH} 7.0$ ), $10 \mathrm{mM}$ glutathione and oxidized glutathione (GSSG), $2.4 \mathrm{mM}$ NADPH and enzyme extract. The detection was based on the rate of decrease in the absorbance of NADPH at $340 \mathrm{~nm}$ for $20 \mathrm{~s}$ $\left(E_{340}=6.2 \mathrm{mM}^{-1} \mathrm{~cm}^{-1}\right)$. All of the spectrophotometric analyses were performed using a SHIMADZU UV-2410PC spectrophotometer (Japan).

\section{Determination of Glutathione}

Glutathione contents were determined according to Sgherri and Navari-Izzo (1995). Root tissues (0.3 g) were homogenized in $2 \mathrm{~mL}$ of $6 \%$ metaphosphoricacid containing $2 \mathrm{mM}$ EDTA. The homogenates were then centrifuged at $4^{\circ} \mathrm{C}$ for $10 \mathrm{~min}$ at $14,000 \mathrm{~g}$.
The total GSSG contents were determined using the 5, 5'dithiobis (2-nitrobenzoicacid)-GSSG reductase recycling method. The reaction mixture for total glutathione (GSH+GSSG) consisted of $100 \mathrm{mM}$ phosphate buffer (PH 7.5), $6 \mathrm{mM} \mathrm{5,5}$ 'dithio-bis (2nitrobenzoicacid; DTNB), $0.2 \mathrm{mM} \mathrm{NADPH}$, three units of GR and extract with the principle of monitoring the decomposition of DTNB at $412 \mathrm{~nm}$ for $1 \mathrm{~min}$. For detection of GSSG, GSH in the extract was blocked out by adding 2-ethenylpyridine, and then measured following the same procedure of total glutathione. Reduced glutathione (GSH) content was then calculated by deducting GSSG from total glutathione (GSH+GSSG).

\section{RNA Isolation and RT-PCR}

Plant root samples were ground in liquid nitrogen and the total RNA was isolated according to the manufacturer's protocol using the Trizol reagent (Invitrogen, California, CA, USA). The genomic DNA was removed using the RNeasy Mini Kit (Qiagen, Beijing, China). Total RNA (1 $\mu \mathrm{g})$ was reverse-transcribed using the ReverTra Ace qPCR RT Kit (Toyobo, Japan) following the manufacturer's instructions. Gene-specific primers for the quantitative real time PCR (qRTPCR) were designed based on the mRNA or EST sequences for the corresponding genes as follows: $\mathrm{Cu} / \mathrm{Zn}$-SOD (F: 5' GGCCAATCTTTGACCCTTTA-3', R: 5'-AGTCCAGGAGCA AGTCCAGT-3'), cAPX (F: 5' - TCTGAATTGGGATTTGCTGA3', R: 5'-CGTCTAACGTAGCTGCCAAA-3'), GR1 (F: 5'-TTG GTGGAACGTGTGTTCTT-3'， R: 5'-TCTCATTCACTTCCC ATCCA-3'), CAT1 (F: 5'-TGATCGCGAGAAGATACCTG-3', R: $5^{\prime}$-CTTCCACGTTCATGGACAAC-3'), and GSH1 (F: 5'-CTGC ATTCTGGGTGGGT-3', R: 5'-CTCGGCTACTTCGTTCA-3'); Actin (F: 5'-TGGTCGGAATGGGACAGAAG-3', R: $5^{\prime}-$ CTCAGTCAG-GAGAACAGGGT- $3^{\prime}$ ) was used as an internal control. For the qRT-PCR, the PCR products were amplified in triplicate using the SYBR Green PCR Master Mix (Takara, Tokyo, Japan) in $25 \mu \mathrm{L}$ qRT-PCR reactions in an iCycler iQ ${ }^{\mathrm{TM}}$ 96-well real-time PCR detection system (Bio-Rad, Hercules, CA, USA). The PCR conditions consisted of denaturation at $95^{\circ} \mathrm{C}$ for $3 \mathrm{~min}$ followed by 40 cycles of denaturation at $95^{\circ} \mathrm{C}$ for $30 \mathrm{~s}$, annealing at $58^{\circ} \mathrm{C}$ for $30 \mathrm{~s}$ and extension at $72^{\circ} \mathrm{C}$ for $30 \mathrm{~s}$. The software that was provided with the PCR system was used to calculate the threshold cycle values and quantify the mRNA levels according to Livak and Schmittgen (2001).

\section{Determination of $\mathrm{Zn}$ Content}

Dry samples $(0.10 \mathrm{~g})$ of shoot or root (homogenized and powdered) were digested with a mixture of $\mathrm{HClO}_{4}$ and $\mathrm{HNO}_{3}$ $(\mathrm{v} / \mathrm{v}=1 / 9)$ at $180^{\circ} \mathrm{C}$. The digested colorless liquids were washed three times with distilled water. The liquid was collected and transferred to $50 \mathrm{~mL}$ volumetric flasks and diluted to a constant volume. Total $\mathrm{Zn}$ concentration was analyzed using an atomic absorption spectrophotometer (AA-6300; Shimadzu Co. Kyoto, Japan) as described by Wu et al. (2005).

\section{Statistical Analysis}

At least four independent replicates were used for each determination, and the mean values of all of the data are presented for each treatment. A statistical analysis of the 
bioassays was performed with the SPSS 16.0 statistical software package, and a Tukey's test $(P<0.05)$ was performed to evaluate the treatment effects.

\section{RESULTS}

\section{Dose Effect of ZnO NPs on Growth and Root Viability of Tomato Seedlings}

To assess toxic effect of $\mathrm{ZnO}$ NPs on seedling growth, we first carried out a dose-trial of $\mathrm{ZnO}$ NPs by exposing tomato seedlings to different levels of $\mathrm{ZnO}$ NPs (10, 20, 50, and $100 \mathrm{mg} / \mathrm{L})$ for 15 days. As shown in Figures 1A,B, both fresh biomass and length of shoot and root of tomato seedlings decreased significantly with increasing concentration of $\mathrm{ZnO}$ NPs in growth media. In addition, the root activity also reduced gradually with an increase in the concentration of $\mathrm{ZnO} \mathrm{NP}$ (Figure 1C). To understand potential link between exposure concentration of $\mathrm{ZnO}$ NPs and subsequent growth inhibition, we quantified
$\mathrm{Zn}$ content in tomato seedlings. Data showed that $\mathrm{Zn}$ content consistently increased with increasing concentration of $\mathrm{ZnO}$ NPs in media (Figure 1D), indicating that $\mathrm{Zn}$ has been absorbed by plant from media in a dose-dependent manner that caused substantial growth inhibition in tomato seedlings.

\section{BR Improves Seedling Growth and Biomass Accumulation under ZnO NPs Stress}

To investigate potential role of BR in tomato $\mathrm{ZnO}$ NPs tolerance, we selected two concentrations of $\mathrm{ZnO}$ NPs (10 and $50 \mathrm{mg} / \mathrm{L}$ ) for further study, considering them as low and high dose of $\mathrm{ZnO}$ NPs based on our preliminary experiment. As shown in Figure 2 both low and high concentration of $\mathrm{ZnO}$ NPs caused significant reduction in biomass accumulation accounting for 20.7/11.9 and $55.2 / 46.3 \%$ reduction in shoot/root, respectively (Figure 2A). Likewise, length of shoot and root decreased by 71.6 and $32.3 \%$, respectively, following exposure of seedlings to $50 \mathrm{mg} / \mathrm{L} \mathrm{ZnO} \mathrm{NPs}$ (Figure 2B). In contrast, combined treatment of BR (5 nM) and
A

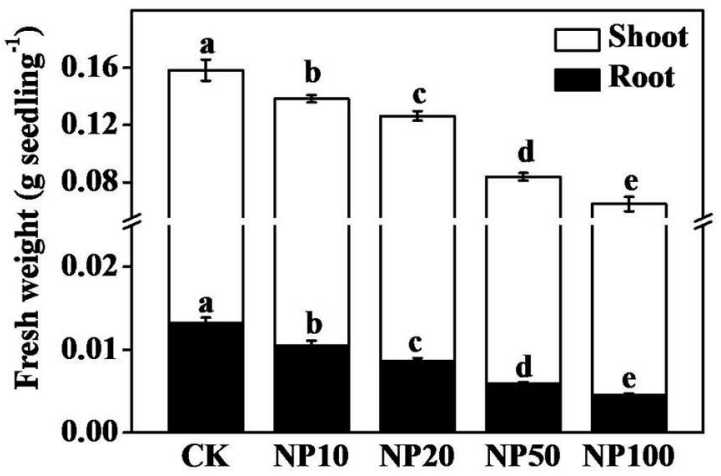

C

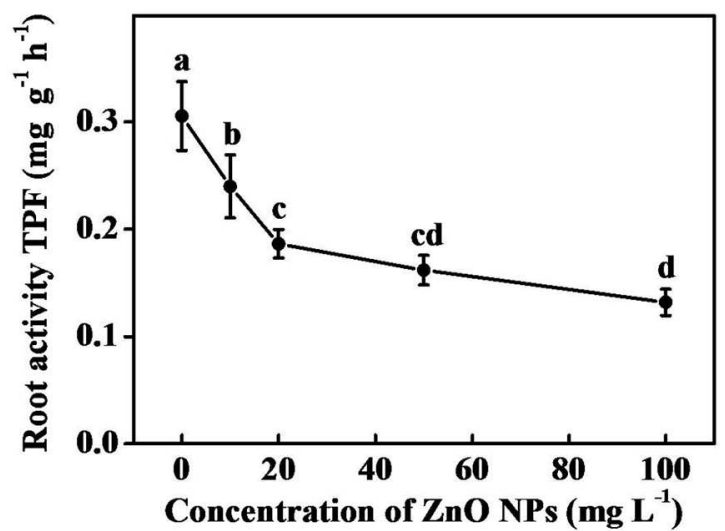

B

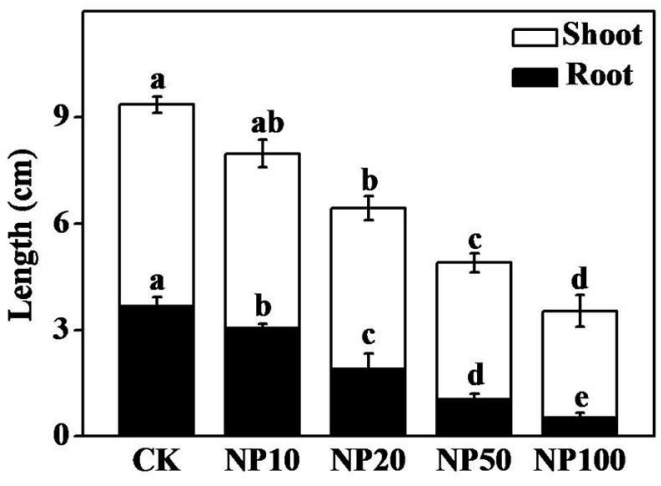

D

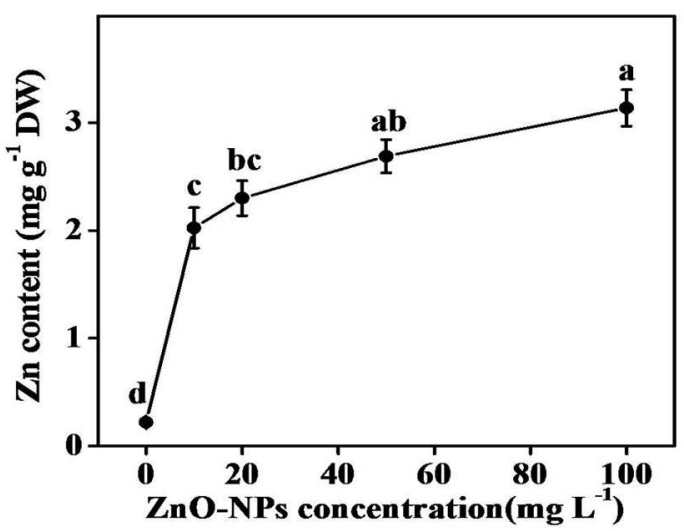

FIGURE 1 | Effect of different concentration of zinc oxide nanoparticles (ZnO NPs) on growth and biomass accumulation in tomato seedlings. (A) Fresh weight, (B) length of shoot and root, (C) root activity, and (D) Zn content in seedlings. Tomato seeds after sprouting were placed on $1 / 2$ MS medium containing graded levels ZnO NPs and cultured for 15 days in tissue culture bottle. Data are the means of four replicates, with standard errors indicated by the vertical bars. Means denoted by the same letter did not significantly differ at a $P<0.05$, according to Tukey's test. CK, control; DW, dry weight; NP10, 10 mg/L ZnO NPs; NP20, 20 mg/L ZnO NPs; NP50, 50 mg/L ZnO NPs; NP100, 100 mg/L ZnO NPs; TPF, triphenyl formazan. 
A

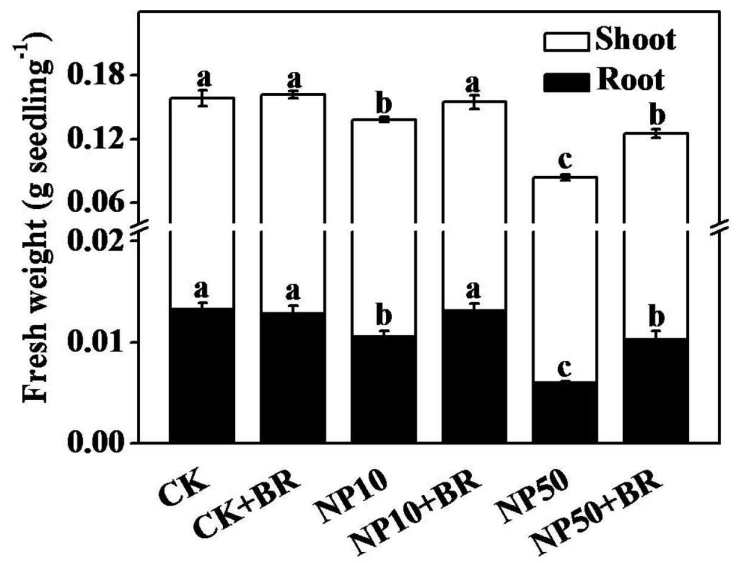

B

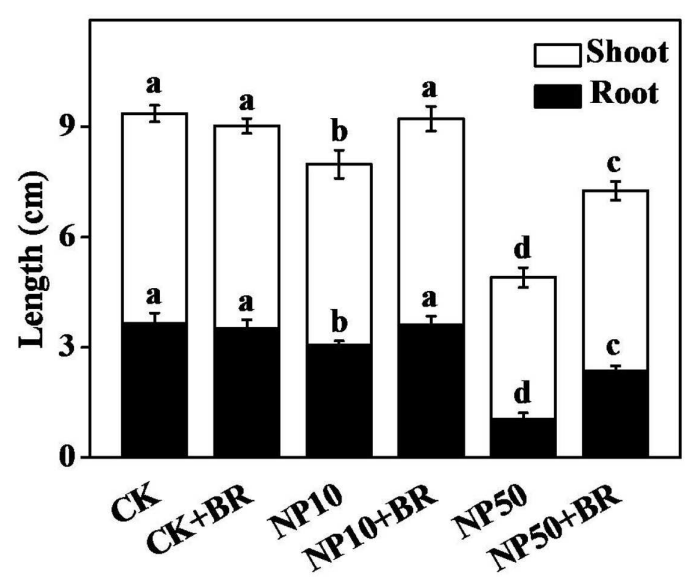

FIGURE 2 | Effect of brassinosteroid on growth and biomass accumulation under zinc oxide nanoparticles (ZnO NPs) stress. (A) Fresh weight, and (B) Length of shoot and root of tomato seedlings after 15 days culture on the $1 / 2$ MS culture medium containing $5 \mathrm{nM}$ 24-epibrassinolide (BR) and/or 10 and 50 mg/L ZnO NPs. The data are the means of four replicates, with standard errors indicated by the vertical bars. Means denoted by the same letter did not significantly differ at a $P<0.05$, according to Tukey's test. NP10, 10 mg/L ZnO NPs; NP50, 50 mg/L ZnO NPs.

ZnO NPs remarkably increased fresh weight and length of shoot and root compared with those of $\mathrm{ZnO}$ NPs (both 10 and $50 \mathrm{mg} / \mathrm{L}$ ) only treatments (Figure 2). For instance, BR treatment with $50 \mathrm{mg} / \mathrm{L} \mathrm{ZnO}$ NPs increased fresh weight and length (shoot/root) by $72.4 / 47.7$ and $126.9 / 26.9 \%$, respectively, compared with those of $50 \mathrm{mg} / \mathrm{L} \mathrm{ZnO} \mathrm{NPs} \mathrm{alone.} \mathrm{These} \mathrm{observations} \mathrm{clearly} \mathrm{indicate}$ that exogenous $\mathrm{BR}(5 \mathrm{nM})$ can alleviate both low and high concentration of $\mathrm{ZnO}$ NPs-induced inhibition of growth and biomass accumulation in tomato seedlings.

\section{BR Minimizes $\mathrm{ZnO}$ NPs-Induced $\mathrm{H}_{2} \mathrm{O}_{2}$ Accumulation and Lipid Peroxidation}

To understand potential mechanism of $\mathrm{ZnO}$ NPs-induced phytotoxicity, we examined $\mathrm{H}_{2} \mathrm{O}_{2}$ accumulation and lipid peroxidation that are often considered as reliable biomarkers of oxidative state. Hitochemical staining with $\mathrm{H}_{2}$ DCF-DA showed that exposure of seedlings to both concentration of $\mathrm{ZnO}$ NPs increased $\mathrm{H}_{2} \mathrm{O}_{2}$ accumulation in roots as evident by remarkably increased green fluorescence compared with that of control (Figure 3A). Biochemical analysis of $\mathrm{H}_{2} \mathrm{O}_{2}$ showed that $\mathrm{H}_{2} \mathrm{O}_{2}$ concentration increased by 27.8 and $66.0 \%$ in 10 and $50 \mathrm{mg} / \mathrm{L}$ $\mathrm{ZnO}$ NPs treatment, respectively, compared with that of control (Figure 3B). When $\mathrm{BR}$ was added with $\mathrm{ZnO} \mathrm{NPs}$ in media, accumulation of $\mathrm{H}_{2} \mathrm{O}_{2}$ was reduced significantly compared with that of $\mathrm{ZnO}$ NPs only treatments (Figure 2B). Interestingly, BR application on non-stressed plants (CK) had no significant effect on $\mathrm{H}_{2} \mathrm{O}_{2}$ accumulation in roots of tomato seedlings (Figure $2 \mathbf{B}$ ). We also quantified the MDA content to evaluate level of lipid peroxidation. In accordance with $\mathrm{H}_{2} \mathrm{O}_{2}$ accumulation, $\mathrm{ZnO}$ NPs (low and high) significantly increased MDA content in roots (Figure 3C). Compared with $\mathrm{ZnO}$ NPs only treatments, MDA content was remarkably decreased when $\mathrm{BR}$ was added with $\mathrm{ZnO}$ NPs in media, indicating that BR could efficiently minimize lipid peroxidation by lowering ROS accumulation in roots.

\section{BR Improves Antioxidant Enzyme Activities and Redox Homeostasis under ZnO NPs Stress}

Responses of antioxidant enzymes to individual and combined treatment of BR and ZnO NPs have been shown in Figure 4. Among four analyzed antioxidant enzymes such as SOD, CAT, APX, and GR, $10 \mathrm{mg} / \mathrm{L} \mathrm{ZnO} \mathrm{NPs} \mathrm{increased} \mathrm{activities} \mathrm{of} \mathrm{CAT}$ and GR only, while $50 \mathrm{mg} / \mathrm{L} \mathrm{ZnO}$ NPs significantly induced activities of all four enzymes. For instance, activities of SOD, CAT, APX, and GR increased by $67.4,85.8,40.3$, and $747.1 \%$ following exposure of seedling to $50 \mathrm{mg} / \mathrm{L} \mathrm{ZnO} \mathrm{NPs} \mathrm{for} 15$ days. Although BR treatment alone increased activity of GR only, combined treatment of BR and ZnO NPs remarkably increased activities of all four enzymes, compared with that of ZnO NPs (low and high) only treatments. These observations further confirm that alleviation of $\mathrm{ZnO}$ NPs-induced oxidative stress, is closely associated with enhancement of antioxidant enzyme activity by BR.

In addition, we assessed cellular redox state by analyzing GSH and GSSG contents, and comparing their ratio under different treatments in roots. Compared with the control, high concentration of ZnO NPs increased the contents of GSH, GSSG and GSH+GSSG, but decreased the GSH:GSSG ratio, whereas low concentration of $\mathrm{ZnO}$ NPs also increased contents of GSH and GSSG but had no significant effect on the GSH:GSSG ratio. Interestingly, combined treatment with $\mathrm{BR}$ and $\mathrm{ZnO}$ NPs significantly increased GSH, but decreased GSSG content, resulting in an increased GSH:GSSG ratio compared with that of $\mathrm{ZnO}$ NPs only treatments (both low and high). For example, compared with $50 \mathrm{mg} / \mathrm{L} \mathrm{ZnO}$ NPs only treatment, combined treatment of $\mathrm{BR}$ and $\mathrm{ZnO}$ NPs increased $\mathrm{GSH}$ by $31.9 \%$, but decreased GSSG by $48.5 \%$ which eventually increased GSH:GSSG ratio by $156.3 \%$. BR only treatment also significantly increased GSH (32.0\%) and decreased GSSG (15.0\%), which ultimately increased the GSH:GSSG ratio (55.4\%) compared with the 


\section{A}
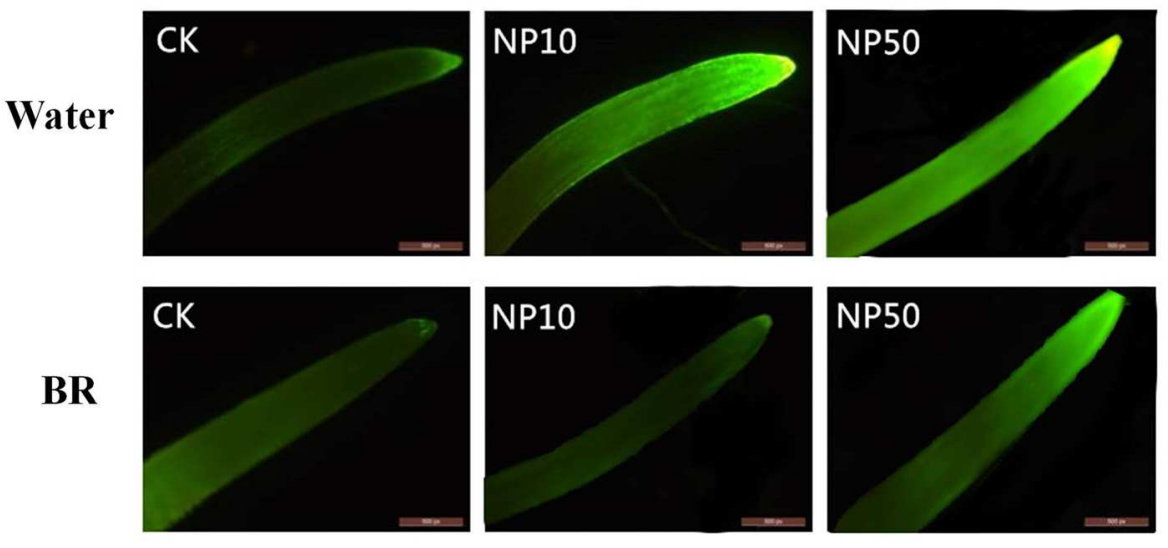

B

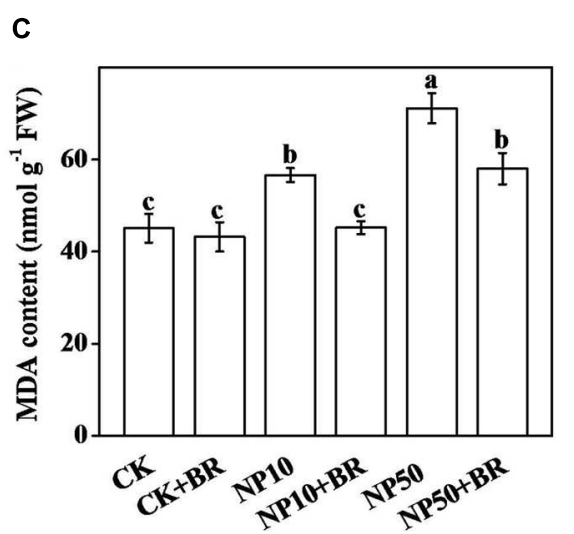

FIGURE $3 \mid \mathrm{H}_{2} \mathrm{O}_{2}$ accumulation and lipid peroxidation in response to brassinosteroid and/or zinc oxide nanoparticles (ZnO NPs) treatments. (A) In situ detection of $\mathrm{H}_{2} \mathrm{O}_{2}$ using H2DCF-DA staining, Bar $=200 \mu \mathrm{m}$, (B) $\mathrm{H}_{2} \mathrm{O}_{2}$ contents, and $\mathbf{( C )}$ malondialdehyde (MDA) contents in seedling roots after 15 days culture in the $1 / 2$ MS medium containing 10 and $50 \mathrm{mg} / \mathrm{L}$ ZnO NPs with or without $5 \mathrm{nM}$ 24-epibrassinolide (BR). The data are the means of four replicates, with standard errors indicated by the vertical bars. Means denoted by the same letter did not significantly differ at a $P<0.05$, according to Tukey's test. CK, control; FW, fresh weight; NP10, 10 mg/L ZnO NPs; NP50, 50 mg/L ZnO NPs.

control. These results clearly indicate that BR-induced alteration in glutathione helped young tomato seedlings to maintain redox homeostasis under $\mathrm{ZnO}$ NPs stress.

\section{BR Upregulates Transcripts of Various Antioxidant-Related Genes under $\mathrm{ZnO}$ NPs Stress}

To assess changes in transcripts of antioxidant genes under $\mathrm{BR}$ and/or $\mathrm{ZnO}$ NPs treatment, we assayed the expression of $\mathrm{Cu} / \mathrm{ZnSOD}, \mathrm{CAT1}$, GSH1, and GR1 genes in tomato roots. The transcript levels of $C u / Z n S O D, C A T 1, G S H 1$, and GR1 genes were all induced by both low and high $\mathrm{ZnO}$ NPs treatments (Figure 6). For example, $50 \mathrm{mg} / \mathrm{L} \mathrm{ZnO} \mathrm{NPs} \mathrm{treatment}$ upregulated transcripts of $\mathrm{Cu} / \mathrm{ZnSOD}, \mathrm{CAT1}, \mathrm{GSH1}$, and GR1 genes by approximately $1.5,2.5,1.8$, and 1.9-fold, respectively. Importantly, addition of BR in media further increased the transcript levels of those genes. Thus, highest levels of transcript abundance were observed when seedlings were treated with BR and $50 \mathrm{mg} / \mathrm{L} \mathrm{ZnO}$ NPs. BR-only treatment also upregulated transcripts of all four genes over control treatment, indicating a positive regulatory role of $\mathrm{BR}$ in transcription of those antioxidant-related genes.

\section{BR Reduces Zn Accumulation under ZnO NPs Stress}

To investigate whether BR-induced alleviation of ZnO NPs stress is also associated with changes in $\mathrm{Zn}$ accumulation, we quantified $\mathrm{Zn}$ content in shoot and root of tomato seedlings following exposure of seedlings to different treatments for 15 days. $\mathrm{Zn}$ accumulation in root was much higher than that in shoot under all treatments. In addition, $\mathrm{Zn}$ contents in shoot and root of BR only treated seedlings were not different from that of CK seedlings. However, exposure of seedlings to low and high $\mathrm{ZnO}$ NPs significantly increased $\mathrm{Zn}$ content both in shoot and root (Figure 7). The accumulation of $\mathrm{Zn}$ was very high in $50 \mathrm{mg} / \mathrm{L}$ $\mathrm{ZnO}$ NPs treatment, accounting for approximately 9.9 and 5.2fold higher in shoot and root, respectively, compared with that of CK. Interestingly, $\mathrm{Zn}$ accumulation significantly reduced in roots and shoots when BR was applied with $\mathrm{ZnO}$ NPs. BR treatment decreased $\mathrm{Zn}$ content by 17.9 and $21.4 \%$ in shoots and roots, 
A

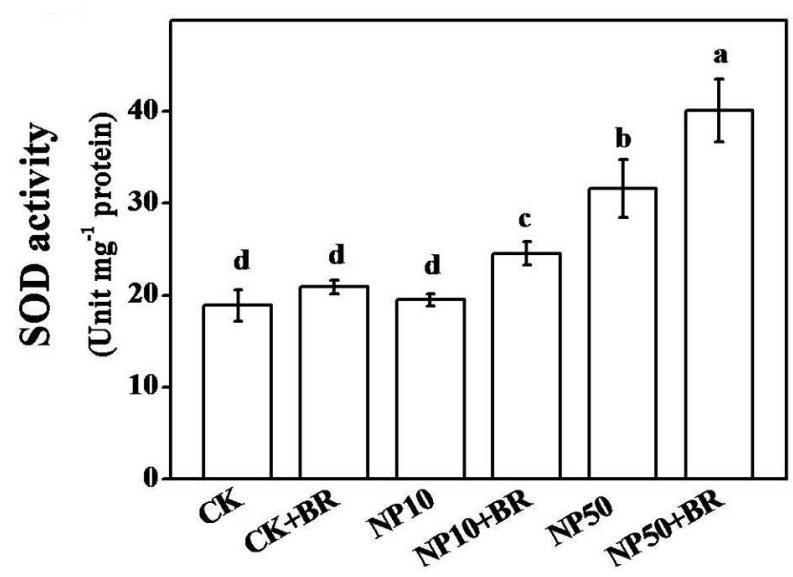

C

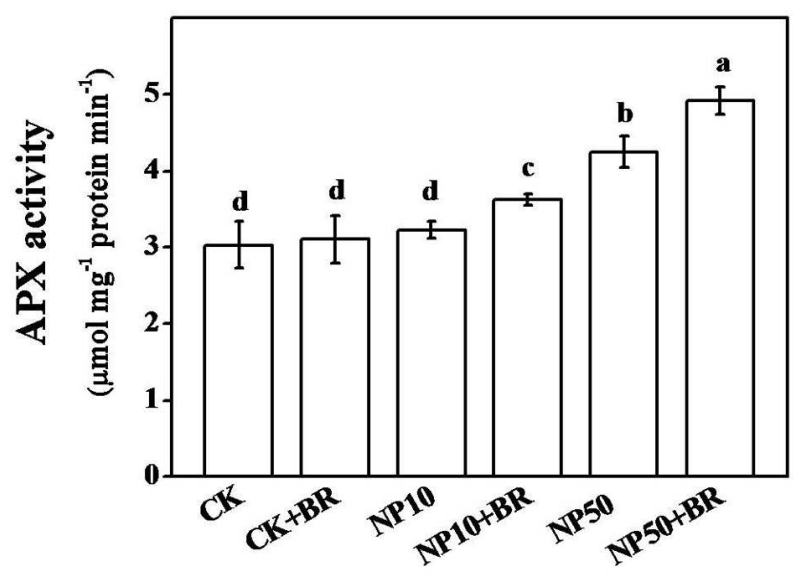

B

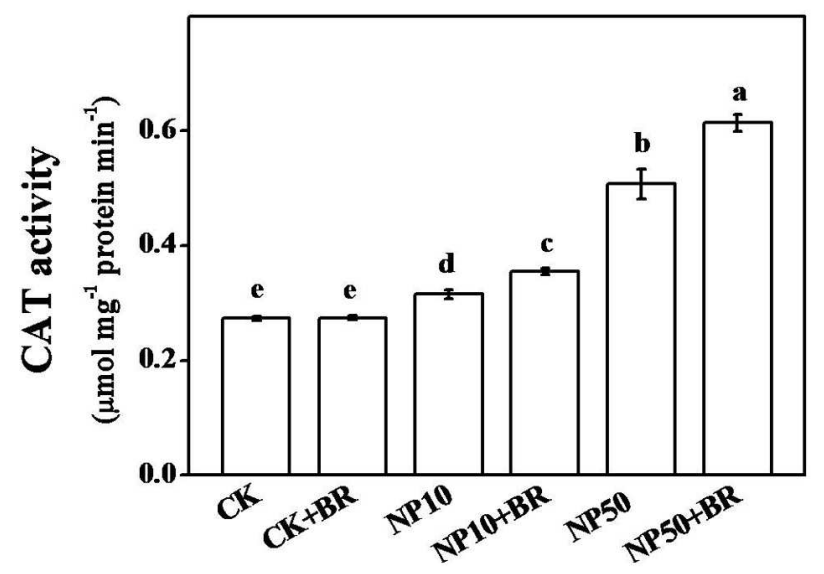

D

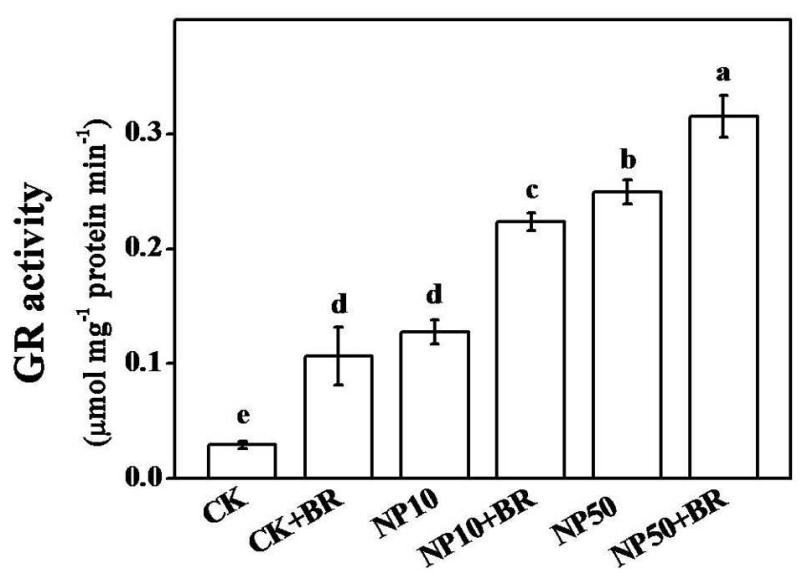

FIGURE 4 | Response of antioxidant enzymes to individual or combined treatment of brassinosteroid and zinc oxide nanoparticles (ZnO NPs). (A) Superoxide dismutase (SOD), (B) Catalase (CAT), (C) Ascorbate peroxidase (APX), and (D) Glutathione reductase (GR) activity in tomato roots after 15 days treatment. Tomato seeds after sprouting were placed on $1 / 2$ MS medium containing $5 \mathrm{nM}$ 24-epibrassinolide (BR) and/or 10 and 50 mg/L ZnO NPs (NP10 and NP50, respectively) and cultured for 15 days before the analysis of activities of antioxidant enzymes. The data shown here are the averages of four replicates, with the standard errors indicated by the vertical bars. The means denoted by the same letter did not significantly differ at a $P<0.05$, according to Tukey's test.

respectively, after exposure to $10 \mathrm{mg} / \mathrm{L} \mathrm{ZnO} \mathrm{NPs}$, while combined treatment of BR with $50 \mathrm{mg} / \mathrm{L} \mathrm{ZnO}$ NPs reduced $\mathrm{Zn}$ content by 25.4 and $17.3 \%$ in shoots and roots, respectively, compared with that in $\mathrm{ZnO}$ NPs-only treatment (Figure 7). These results indicate that alleviation of ZnO NPs-induced oxidative stress by BR was attributed to BR-mediated reduction in $\mathrm{Zn}$ content in shoot and root of tomato seedlings under ZnO NPs stress.

\section{DISCUSSION}

Despite the discrepancy in research findings relating to NPsinduced phytotoxicity, it is now well evident that large scale use of metal-based NPs (MNPs) could appear as a serious threat to crop production and food safety (Chichiriccò and Poma,
2015; Van Aken, 2015). Development of approaches that can alleviate MNPs-induced phytotoxicity may ensure sustainable crop production in the MNPs-polluted marginal lands. In the current study, for the first time, we showed that $\mathrm{ZnO}$ NPs-induced phytotoxicity could be alleviated by exogenous application of BR at optimal concentration ( $5 \mathrm{nM}$ ). BR efficiently ameliorated $\mathrm{ZnO}$ NPs-induced oxidative stress by promoting antioxidant enzyme activities, redox homeostasis and related gene expression (Figures 2-6). In addition, BR decreased $\mathrm{Zn}$ content in both shoot and root of young tomato seedling under $\mathrm{ZnO}$ NPs stress (Figure 7). This study provides promising data that support beneficial role of BR in enhancing plant tolerance to $\mathrm{ZnO}$ NPs stress and ensuring food safety.

Phytotoxicity of MNPs is not only due to their small size, large surface area and intrinsic reactivity that allow them to 


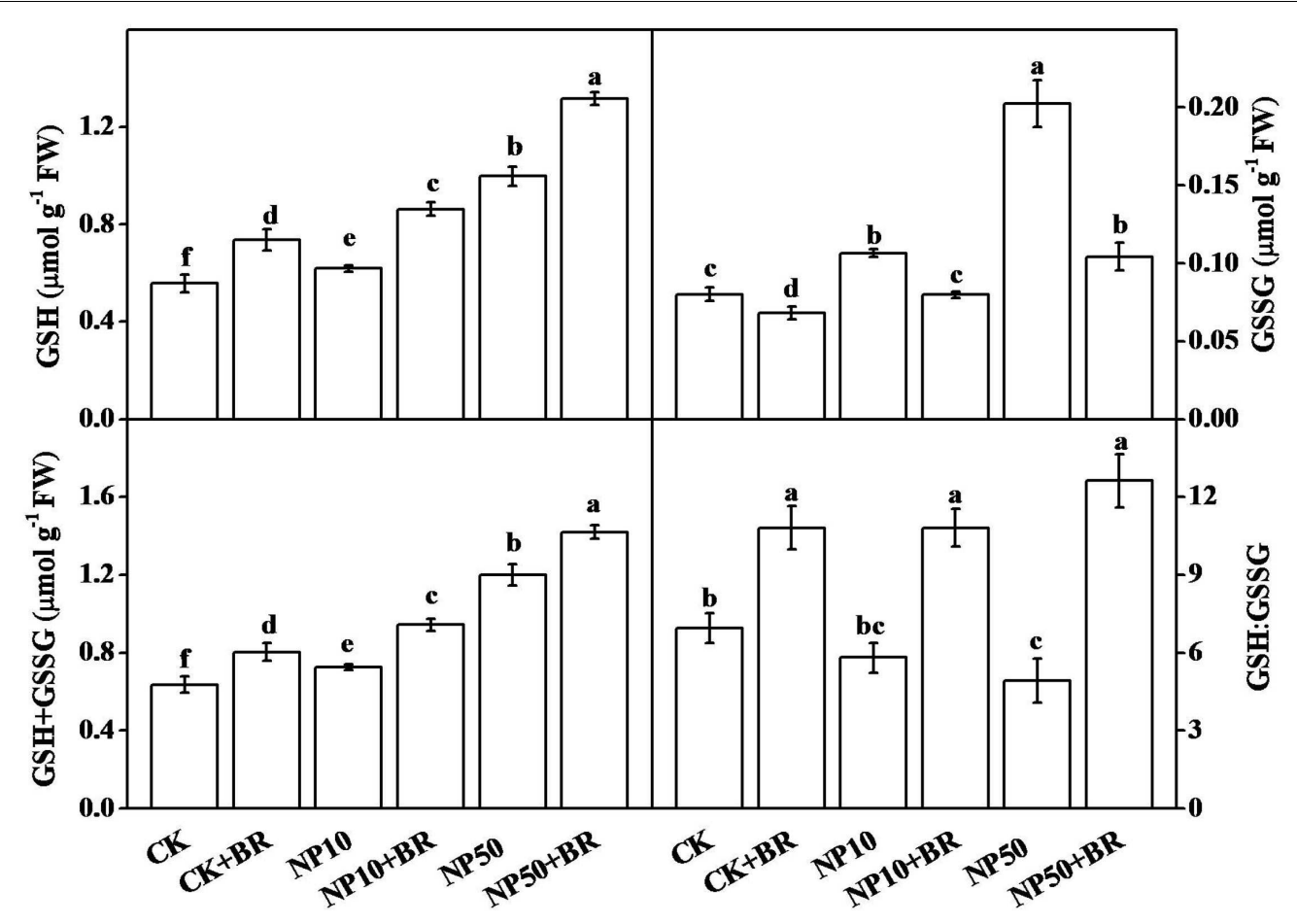

FIGURE 5 | Effects of zinc oxide nanoparticules (ZnO NPs) and brassinosteroid on glutathione content and redox homeostasis in roots of tomato seedlings. Tomato seeds after sprouting were placed on $1 / 2$ MS medium containing 5 nM 24-epibrassinolide (BR) and/or 10 and 50 mg/L ZnO NPs (NP10 and NP50, respectively) and cultured for 15 days before the analysis of glutathione content. The data shown here are the averages of four replicates, with the standard errors indicated by the vertical bars. The means denoted by the same letter did not significantly differ at a $P<0.05$, according to Tukey's test. GSH, reduced glutathione; GSSG, oxidized glutathione; GSH+GSSG, total glutathione; GSH:GSSG, ratio between reduced and oxidized glutathione.

interact with biological macromolecules (protein and nucleic acid), but also associated with the metal constituents. Prior research has shown that MNPs dissolve quickly releasing metal ions that induce production of ROS such as $\mathrm{H}_{2} \mathrm{O}_{2}$ and ${ }^{\bullet} \mathrm{OH}$ that are capable to damage vital biomolecules including protein, lipids and DNA (Van Aken, 2015). It is anticipated that $\mathrm{ZnO}$ NPs firstly dissolve and then penetrate the cells in the form of $\mathrm{Zn}^{2+}$ (Chichiriccò and Poma, 2015). Once they penetrate into the plant, deleterious effects may be produced. In the present study, $\mathrm{ZnO}$ NPs exerted a dose-dependent inhibitory effect on tomato growth and biomass accumulation, which was closely associated with accumulation of $\mathrm{Zn}$ in plant tissue with increasing concentration of $\mathrm{ZnO}$ NPs in culture media (Figure 1). ZnO NPs have been shown to reduce root length in corn and cucumber (Zhang et al., 2015), which supports our current observation. It is quite plausible that $\mathrm{ZnO} \mathrm{NPs}$ inhibit seedling growth concomitantly by directly interacting with biomolecules and also inducing ROS production (Chichiriccò and Poma, 2015). Excessive production of ROS eventually causes an oxidative stress that may lead to cell death (Baxter et al., 2014). To confirm occurrence of oxidative stress upon $\mathrm{ZnO}$ NPs treatment, we visualized $\mathrm{H}_{2} \mathrm{O}_{2}$ accumulation and biochemically quantified concentration of $\mathrm{H}_{2} \mathrm{O}_{2}$ and $\mathrm{MDA}$, an important marker of lipid peroxidation (Figure 3). Our data clearly indicate that ZnO NPsinduced growth inhibition was associated with excessive levels $\mathrm{H}_{2} \mathrm{O}_{2}$ and MDA. These results are well in accordance with prior reports that showed that MNPs caused plant growth inhibition by inducing oxidative stress in a range of plant species (Dietz and Herth, 2011; Chichiriccò and Poma, 2015).

Besides regulating plant growth and development, phytohormone BR plays a critical role in controlling plant stress responses (Divi et al., 2016). These properties have established BR as a promising plant hormone that could stabilize crop yield under various abiotic stresses such as drought, salinity, extreme temperatures, heavy metals, and organic pollutant stress (Ahammed et al., 2014; Divi et al., 2016). We previously showed that BRs confer $\mathrm{Cd}$ tolerance by reducing $\mathrm{H}_{2} \mathrm{O}_{2}$ accumulation and activating antioxidant potential in tomato (Ahammed et al., 2013). Recently, Ramakrishna and Rao (2015) reported that foliar application of $\mathrm{BR}$ mitigates $\mathrm{ZnO}$-induced oxidative stress in radish. In conformity with earlier reports, here we observed that addition of $5 \mathrm{nM}$ BR in $1 / 2 \mathrm{MS}$ media remarkably increased tomato seedling growth and biomass accumulation under 10 and $50 \mathrm{mg} / \mathrm{L} \mathrm{ZnO} \mathrm{NPs} \mathrm{stress} \mathrm{(Figure} \mathrm{2).} \mathrm{This} \mathrm{response} \mathrm{of} \mathrm{BR}$ was highly dose-specific as we noticed an inhibitory effect of $\mathrm{BR}$ on seedling growth when applied at $10 \mathrm{nM}$ concentration (data not shown). It is worth mentioning that BRs-regulated plant stress tolerance in a highly dose-dependent manner that greatly differs across the plant species as well as growth stage and tissue type (Ahammed et al., 2014). In tomato, exogenous BR application or endogenous BR level upregulation by overexpression of $\mathrm{BR}$ biosynthetic gene $D W A R F$ results 


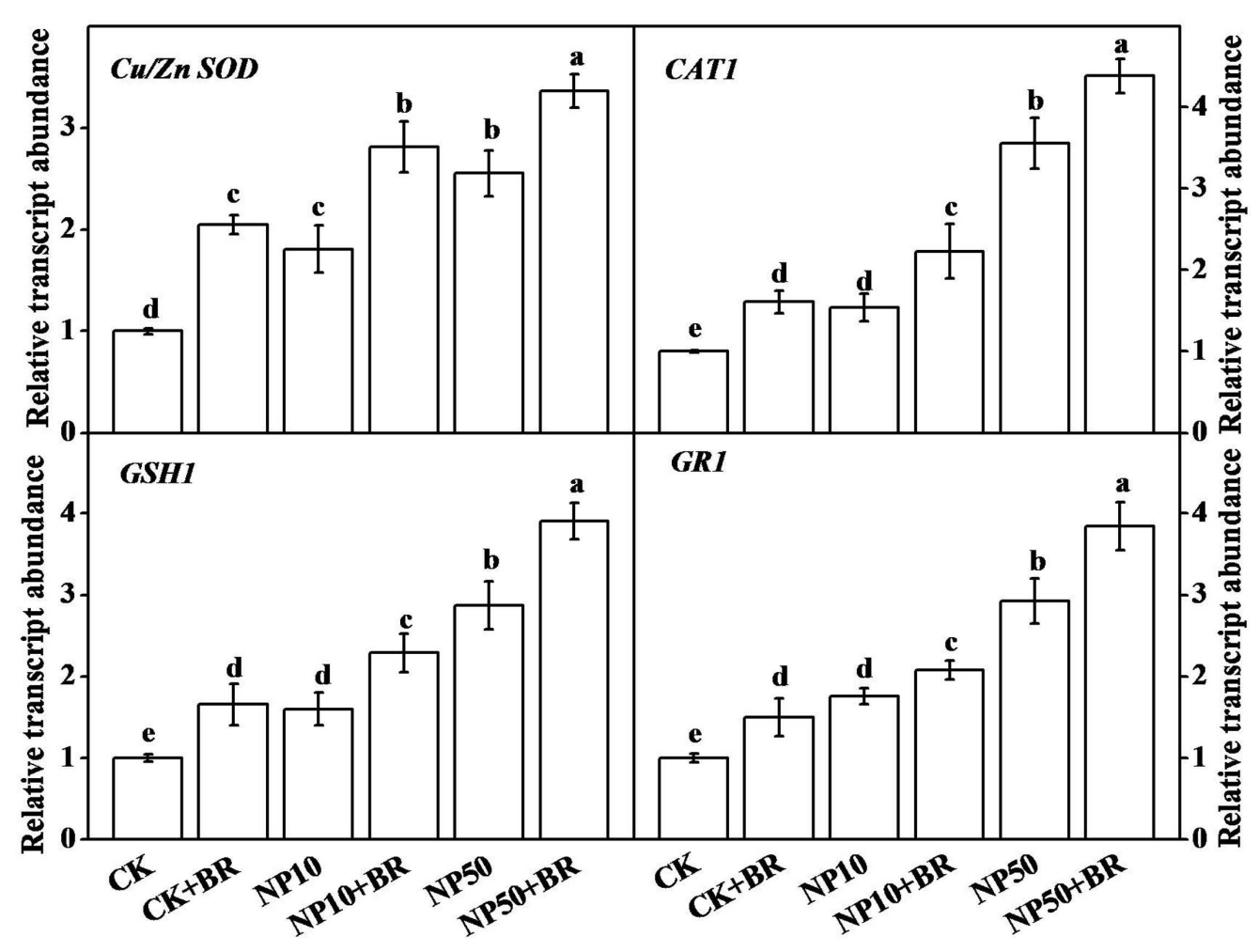

FIGURE 6 | Expression of antioxidant genes in tomato roots as influence by brassinosteroid and zinc oxide nanoparticles (ZnO NPs). The expression of genes was analyzed by qRT-PCR after 15 days treatment using gene-specific primer pairs. The data shown here are the averages of four replicates, with the standard errors indicated by the vertical bars. The means denoted by the same letter did not significantly differ at a $P<0.05$, according to Tukey's test. BR, 5 nM 24-epibrassinolide; CK, control; NP10, 10 mg/L ZnO NPs; NP50, 50 mg/L ZnO NPs.

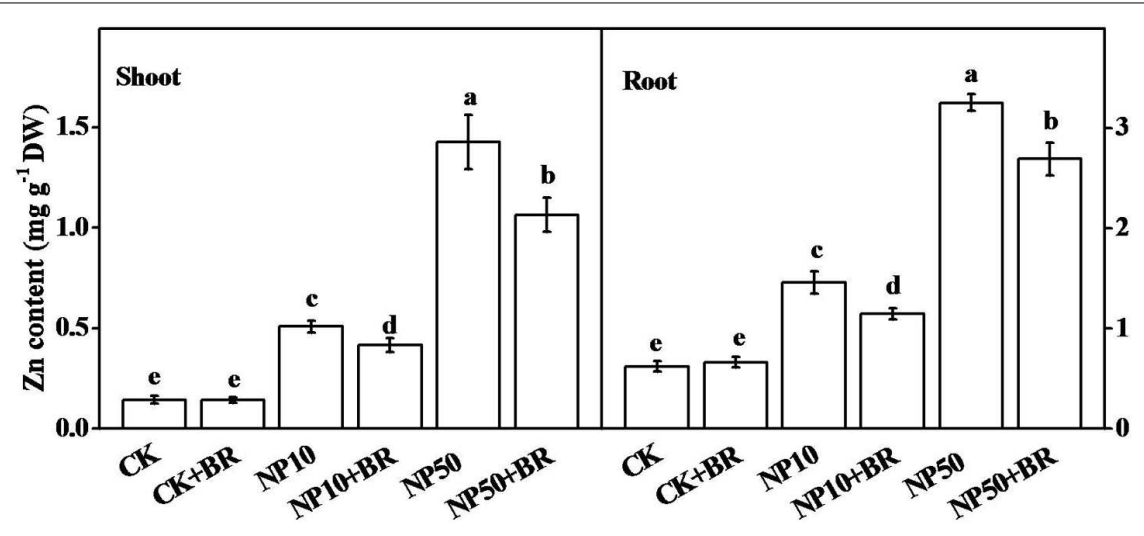

FIGURE 7 | Interactive effect of zinc oxide nanoparticles (ZnO NPs) and brassinosteroid on accumulation of Zinc in tomato seedlings. Tomato seeds after sprouting were placed on $1 / 2$ MS medium containing 5 nM 24-epibrassinolide (BR) and/or 10 and 50 mg/L ZnO NPs (NP10 and NP50, respectively) and cultured for 15 days before the analysis of Zn content. The data shown here are the averages of four replicates, with the standard errors indicated by the vertical bars. The means denoted by the same letter did not significantly differ at a $P<0.05$, according to Tukey's test.

in improved plant growth (Li et al., 2015). BR stimulates cell division, elongation and development that are closely associated with BR-promoted plant growth (Ahammed et al., 2014; Divi et al., 2016).

Reactive oxygen species play a dual role in plant system; on one hand they serve as a critical signaling molecule and on the other hand they cause toxicity when accumulated at high level especially under stress (Baxter et al., 2014). Our prior study showed that $\mathrm{H}_{2} \mathrm{O}_{2}$, a typical ROS, is intricately involved in BRmediated stress response (Xia et al., 2009). BR triggers transient accumulation of NADPH-based $\mathrm{H}_{2} \mathrm{O}_{2}$ which in turn stimulates antioxidant potential by inducing transcript of antioxidant 
biosynthetic genes (Cui et al., 2011; Nie et al., 2013; Divi et al., 2016). BR-promoted activities of antioxidant enzymes eventually increase ROS scavenging and thus minimizing oxidative stress under stress (Cui et al., 2011). In the current study, BR treatment significantly reduced accumulation of harmful level of $\mathrm{H}_{2} \mathrm{O}_{2}$ under $\mathrm{ZnO}$ NPs stress (Figure $\mathbf{3 A}$ ). In addition to reduction in $\mathrm{H}_{2} \mathrm{O}_{2}$ content, BR-mediated decrease in MDA content revealed that $\mathrm{BR}$ efficiently alleviated $\mathrm{ZnO}$ NPs-induced oxidative stress in tomato seedlings (Figure 3C). This observation was further confirmed by analysis of antioxidant enzyme activities. Supplementation of BR remarkably increased activities of SOD, CAT, APX, and GR under ZnO NPs stress. In plant cells, SOD can rapidly convert $\mathrm{O}_{2}^{\bullet-}$ to $\mathrm{H}_{2} \mathrm{O}_{2}$. Once levels of $\mathrm{H}_{2} \mathrm{O}_{2}$ are high due to catalytic activity of SOD or stress induction, some other antioxidative pathways may be activated to diminish excessive $\mathrm{H}_{2} \mathrm{O}_{2}$. CAT is a common antioxidant enzyme that converts $\mathrm{H}_{2} \mathrm{O}_{2}$ to $\mathrm{H}_{2} \mathrm{O}$ and $\mathrm{O}_{2}$. Besides, APX can also convert $\mathrm{H}_{2} \mathrm{O}_{2}$ to $\mathrm{H}_{2} \mathrm{O}$ involving ascorbate-glutathione cycle. In addition, $\mathrm{GSH}$ is a key antioxidant molecule and can directly decompose $\mathrm{H}_{2} \mathrm{O}_{2}$ by producing oxidant glutathione (GSSG), which is then reduced to GSH by GR (Ma et al., 2015). Our results are in conformity with many earlier reports that showed that application of BR conferred stress tolerance by inducing antioxidant enzyme activities in different plant species (Ramakrishna and Rao, 2012, 2015, p. 29; Ahammed et al., 2013, 2014; Vardhini and Anjum, 2015).

Maintenance of cellular redox homeostasis is critical for normal metabolic processes in plants (Baxter et al., 2014). Under MNPs stress, redox balance is disrupted due to over accumulation of ROS (Chichiriccò and Poma, 2015; Van Aken, 2015). In the current study, GSH:GSSG ratio was significantly decreased under $50 \mathrm{mg} / \mathrm{L} \mathrm{ZnO}$ NPs stress, indicating an oxidized redox state which is considered harmful for plants. Ag NPs stress also reduced plant biomass but increased accumulation of oxidized glutathione (GSSG, indicative of oxidative stress) in Triticum aestivum (Van Aken, 2015). Treatment with BR decreased GSSG content under $\mathrm{ZnO}$ NPs stress, which helped young tomato seedlings to maintain a high GSH:GSSG ratio that was almost equivalent to non-stressed seedlings (Figure 5). These results are in agreement with earlier reports that showed that BRs could maintain redox homeostasis under $\mathrm{Zn}$ and $\mathrm{Cd}$ stress in radish and tomato, respectively (Ramakrishna and Rao, 2012; Ahammed et al., 2013).

Brassinosteroid enhances plant tolerance to broad-range stresses, while such enhancement in stress tolerance is correlated with higher expression of genes that are considered as stress markers (Divi et al., 2016). Transcription of antioxidant related genes are generally upregulated under stress (Baxter et al., 2014). To study the molecular bases of the toxicity of ZnO NPs and alleviatory role of $\mathrm{BR}$, we studied expression of antioxidant related genes by using qRT-PCR. In this study, transcripts of $\mathrm{Cu} / \mathrm{Zn}$ SOD, GSH1, CAT1, and GR1 were induced by $\mathrm{ZnO}$ NPs treatment and BR co-application further upregulated their transcript abundance in roots of tomato seedlings. This observation is in line with previous reports, which showed that BR could upregulate expression of various antioxidant related genes in rice and tomato under $\mathrm{Cr}$ and $\mathrm{Cd}$ stress, respectively (Ahammed et al., 2013; Sharma et al., 2016).

Given that plants are vital components of the food chain, its interaction with NPs essentially have major implications in understanding of environment and public health (Van Aken, 2015). Concerning the connection between BR and food safety, a number of studies have shown that BR could reduce heavy metal accumulation in plants (Ahammed et al., 2013, 2014; Ramakrishna and Rao, 2015; Sharma et al., 2016). In conformity with earlier reports, here we found that BR treatment caused a significant reduction in $\mathrm{Zn}$ accumulation under $\mathrm{ZnO}$ NPs stress in tomato seedling (Figure 7). This implies that BR could also reduce accumulation of metals from MNPs, and thus suggesting a potential implication of BR in ensuring food safety in MNPspolluted areas. In a recent study, (Chen et al., 2015) showed that NO could alleviate ZnO NPs-induced phytotoxicity by reducing $\mathrm{Zn}$ accumulation in rice seedlings. $\mathrm{NO}$ is an important signaling molecule that enhances plant tolerance to various environmental stresses. In our earlier study, we found that NO is involved in BRinduced oxidative stress tolerance in cucumber (Cui et al., 2011). Therefore, BR-induced alleviation of $\mathrm{ZnO}$ NPs phytotoxicity might also be mediated by regulation of NO (Cui et al., 2011; Chen et al., 2015).

Taken together, this study showed that $\mathrm{ZnO}$ NPs induced oxidative stress by increasing accumulation of $\mathrm{Zn}$ in plant tissue, which in turn reduced plant growth and biomass accumulation possibly by affecting vital cellular processes. In contrast, treatment with $\mathrm{BR}$ alleviated $\mathrm{ZnO}$ NPs-induced growth inhibition and oxidative stress by improving antioxidant potential and redox homeostasis. The alleviation of ZnO NPsinduced oxidative stress by $\mathrm{BR}$, was closely associated with upregulation of transcripts of antioxidant genes. BR-induced reduction in $\mathrm{Zn}$ content under $\mathrm{ZnO}$ NPs stress, provides new prospect for stabilizing crop yield and quality in MNPscontaminated land. To our knowledge, this study provides the first evidence that $\mathrm{BR}$ could alleviate $\mathrm{ZnO}$ NPs-induced phytotoxicity in tomato. However, further study involving advanced genetic and molecular tools may help to better understand the detailed molecular mechanisms of BR-induced ZnO NPs tolerance in plants.

\section{AUTHOR CONTRIBUTIONS}

JZ, ML, GA, and JY planned and designed the research. ML, $\mathrm{CL}, \mathrm{XB}, \mathrm{CH}$, and $\mathrm{HY}$ performed experiments. JZ, ML, and GA analyzed data. JZ, ML, and GA wrote the manuscript.

\section{ACKNOWLEDGMENT}

This work was supported by the National Natural Science Foundation of China (31550110201, 31430076, 31401877), the Geological Exploration Foundation of Zhejiang Province, China (2014002-02) and the China Postdoctoral Science Foundation (517000-X91512). 


\section{REFERENCES}

Ahammed, G., Xia, X., Li, X., Shi, K., Yu, J., and Zhou, Y. (2014). Role of brassinosteroid in plant adaptation to abiotic stresses and its interplay with other hormones. Curr. Protein Pept. Sci. 16, 462-473. doi: $10.2174 / 1389203716666150330141427$

Ahammed, G. J., Choudhary, S. P., Chen, S., Xia, X., Shi, K., Zhou, Y., et al. (2013). Role of brassinosteroids in alleviation of phenanthrenecadmium co-contamination-induced photosynthetic inhibition and oxidative stress in tomato. J. Exp. Bot. 64, 199-213. doi: 10.1093/jxb/ ers 323

Ali, B., Hasan, S. A., Hayat, S., Hayat, Q., Yadav, S., Fariduddin, Q., et al. (2008). A role for brassinosteroids in the amelioration of aluminium stress through antioxidant system in mung bean (Vigna radiata L. Wilczek). Environ. Exp. Bot. 62, 153-159. doi: 10.1016/j.envexpbot.2007.07.014

Baxter, A., Mittler, R., and Suzuki, N. (2014). ROS as key players in plant stress signalling. J. Exp. Bot. 65, 1229-1240. doi: 10.1093/jxb/ ert375

Cakmak, I., and Marschner, H. (1992). Magnesium deficiency and high light intensity enhance activities of superoxide dismutase, ascorbate peroxidase, and glutathione reductase in bean leaves. Plant Physiol. 98, 1222-1227. doi: 10.1104/pp.98.4.1222

Chen, J., Liu, X., Wang, C., Yin, S. S., Li, X. L., Hu, W. J., et al. (2015). Nitric oxide ameliorates zinc oxide nanoparticles-induced phytotoxicity in rice seedlings. J. Hazard. Mater. 297, 173-182. doi: 10.1016/j.jhazmat.2015. 04.077

Chichiriccò, G., and Poma, A. (2015). Penetration and toxicity of nanomaterials in higher plants. Nanomaterials 5, 851-873. doi: 10.3390/nano5 020851

Cui, J.-X., Zhou, Y.-H., Ding, J.-G., Xia, X.-J., Shi, I., Chen, S.-C., et al. (2011). Role of nitric oxide in hydrogen peroxide-dependent induction of abiotic stress tolerance by brassinosteroids in cucumber. Plant Cell Environ. 34, 347-358. doi: 10.1111/j.1365-3040.2010.02248.x

Dietz, K. J., and Herth, S. (2011). Plant nanotoxicology. Trends Plant Sci. 16, 582-589. doi: 10.1016/j.tplants.2011.08.003

Dimkpa, C. O., Latta, D. E., Mclean, J. E., Britt, D. W., Boyanov, M. I., and Anderson, A. J. (2013). Fate of $\mathrm{CuO}$ and $\mathrm{ZnO}$ nano- and microparticles in the plant environment. Environ. Sci. Technol. 47, 4734-4742. doi: 10.1021/es304736y

Divi, U. K., Rahman, T., and Krishna, P. (2016). Gene expression and functional analyses in brassinosteroid-mediated stress tolerance. Plant. Biotechnol. J. 14, 419-432. doi: 10.1111/pbi.12396

Fosmire, G. J. (1990). Zinc toxicity. Am. J. Clin. Nutr. 51, 225-227.

Foyer, C. H., and Halliwell, B. (1976). The presence of glutathione and glutathione reductase in chloroplasts: a proposed role in ascorbic acid metabolism. Planta 133, 21-25. doi: 10.1007/BF00386001

He, L., Liu, Y., Mustapha, A., and Lin, M. (2011). Antifungal activity of zinc oxide nanoparticles against Botrytis cinerea and Penicillium expansum. Microbiol. Res. 166, 207-215. doi: 10.1016/j.micres.2010.03.003

Hernandez-Viezcas, J., Castillo-Michel, H., Servin, A., Peralta-Videa, J., and Gardea-Torresdey, J. (2011). Spectroscopic verification of zinc absorption and distribution in the desert plant Prosopis juliflora-velutina (velvet mesquite) treated with $\mathrm{ZnO}$ nanoparticles. Chem. Eng. J. 170, 346-352. doi: 10.1016/j.cej.2010.12.021

Hodges, D. M., Delong, J. M., Forney, C. F., and Prange, R. K. (1999). Improving the thiobarbituric acid-reactive-substances assay for estimating lipid peroxidation in plant tissues containing anthocyanin and other interfering compounds. Planta 207, 604-611. doi: 10.1007/s004250050524

Hoffmann, M. R., Martin, S. T., Choi, W., and Bahnemann, D. W. (1995). Environmental applications of semiconductor photocatalysis. Chem. Rev. 95, 69-96. doi: 10.1021/cr00033a004

Islam, E., Yang, X., Li, T., Liu, D., Jin, X., and Meng, F. (2007). Effect of $\mathrm{Pb}$ toxicity on root morphology, physiology and ultrastructure in the two ecotypes of Elsholtzia argyi. J. Hazard. Mater. 147, 806-816. doi: 10.1016/j.jhazmat.2007.01.117

Li, X. J., Chen, X. J., Guo, X., Yin, L. L., Ahammed, G. J., Xu, C. J., et al. (2015). DWARF overexpression induces alteration in phytohormone homeostasis, development, architecture and carotenoid accumulation in tomato. Plant Biotechnol. J. 14, 1021-1033. doi: 10.1111/pbi.12474

Lin, D., and Xing, B. (2008). Root uptake and phytotoxicity of $\mathrm{ZnO}$ nanoparticles. Environ. Sci. Technol. 42, 5580-5585. doi: 10.1021/es80 $0422 \mathrm{x}$

Livak, K. J., and Schmittgen, T. D. (2001). Analysis of relative gene expression data using real-time quantitative PCR and the 2- $\Delta \Delta$ CT method. Methods 25, 402-408. doi: 10.1006/meth.2001.1262

Ma, C., White, J. C., Dhankher, O. P., and Xing, B. (2015). Metal-based nanotoxicity and detoxification pathways in higher plants. Environ. Sci. Technol. 49, 7109-7122. doi: 10.1021/acs.est.5b00685

Ma, H., Williams, P. L., and Diamond, S. A. (2013). Ecotoxicity of manufactured $\mathrm{ZnO}$ nanoparticles-a review. Environ. Pollut. 172, 76-85. doi: 10.1016/j.envpol.2012.08.011

Nakano, Y., and Asada, K. (1981). Hydrogen peroxide is scavenged by ascorbate-specific peroxidase in spinach chloroplasts. Plant Cell Physiol. 22, 867-880.

Nie, W. F., Wang, M. M., Xia, X. J., Zhou, Y. H., Shi, K., Chen, Z., et al. (2013). Silencing of tomato RBOH1 and MPK2 abolishes brassinosteroid-induced H2O2 generation and stress tolerance. Plant Cell Environ. 36, 789-803. doi: 10.1111/pce.12014

Pokhrel, L. R., and Dubey, B. (2013). Evaluation of developmental responses of two crop plants exposed to silver and zinc oxide nanoparticles. Sci. Total Environ. 452, 321-332. doi: 10.1016/j.scitotenv.2013. 02.059

Ramakrishna, B., and Rao, S. S. (2012). 24-Epibrassinolide maintains elevated redox state of AsA and GSH in radish (Raphanus sativus L.) seedlings under zinc stress. Act. Physiol. Plant. 35, 1291-1302. doi: 10.1007/s11738-0121168-7

Ramakrishna, B., and Rao, S. S. (2015). Foliar application of brassinosteroids alleviates adverse effects of zinc toxicity in radish (Raphanus sativus L.) plants. Protoplasma 252, 665-677. doi: 10.1007/s00709-0140714-0

Sgherri, C. L. M., and Navari-Izzo, F. (1995). Sunflower seedlings subjected to increasing water deficit stress: oxidative stress and defence mechanisms. Physiol. Plant 93, 25-30. doi: 10.1034/j.1399-3054.1995.93 0105.x

Sharma, P., Kumar, A., and Bhardwaj, R. (2016). Plant steroidal hormone epibrassinolide regulate - Heavy metal stress tolerance in Oryza sativa L. by modulating antioxidant defense expression. Environ. Exp. Bot. 122, 1-9. doi: 10.1016/j.envexpbot.2015.08.005

Stewart, R. R., and Bewley, J. D. (1980). Lipid peroxidation associated with accelerated aging of soybean axes. Plant Physiol. 65, 245-248. doi: 10.1104/pp.65.2.245

Van Aken, B. (2015). Gene expression changes in plants and microorganisms exposed to nanomaterials. Curr. Opin. Biotechnol. 33, 206-219. doi: 10.1016/j.copbio.2015.03.005

Vardhini, B. V., and Anjum, N. A. (2015). Brassinosteroids make plant life easier under abiotic stresses mainly by modulating major components of antioxidant defense system. Front. Environ. Sci. 2:67. doi: 10.3389/fenvs.2014.00067

Willekens, H., Chamnongpol, S., Davey, M., Schraudner, M., Langebartels, C., Vanmontagu, M., et al. (1997). Catalase is a sink for $\mathrm{H} 2 \mathrm{O} 2$ and is indispensable for stress defence in C-3 plants. $E M B O ~ J .16,4806-4816$. doi: 10.1093/emboj/16.16.4806

Wu, F.-B., Dong, J., Qian, Q. Q., and Zhang, G.-P. (2005). Subcellular distribution and chemical form of $\mathrm{Cd}$ and $\mathrm{Cd}-\mathrm{Zn}$ interaction in different barley genotypes. Chemosphere 60, 1437-1446. doi: 10.1016/j.chemosphere.2005. 01.071

Xia, X.-J., Wang, Y.-J., Zhou, Y.-H., Tao, Y., Mao, W.-H., Shi, K., et al. (2009). Reactive oxygen species are involved in brassinosteroid-induced stress tolerance in cucumber. Plant Physiol. 150, 801-814. doi: 10.1104/pp.109. 138230

Yi, C., Yao, K., Cai, S., Li, H., Zhou, J., Xia, X., et al. (2015). High atmospheric carbon dioxide-dependent alleviation of salt stress is linked to RESPIRATORY BURST OXIDASE 1 (RBOH1)-dependent $\mathrm{H} 2 \mathrm{O} 2$ production in tomato (Solanum lycopersicum). J. Exp. Bot. 66, 7391-7404. doi: 10.1093/jxb/ erv 435 
Zhang, R., Zhang, H., Tu, C., Hu, X., Li, L., Luo, Y., et al. (2015). Phytotoxicity of $\mathrm{ZnO}$ nanoparticles and the released $\mathrm{Zn}$ (II) ion to corn (Zea mays L.) and cucumber (Cucumis sativus L.) during germination. Environ. Sci. Pollut. Res. Int. 22, 11109-11117. doi: 10.1007/s11356-015-4325-x

Zheng, N., Wang, Q., and Zheng, D. (2007). Health risk of $\mathrm{Hg}, \mathrm{Pb}, \mathrm{Cd}, \mathrm{Zn}$, and $\mathrm{Cu}$ to the inhabitants around Huludao Zinc Plant in China via consumption of vegetables. Sci. Total Environ. 383, 81-89.

Zhou, J., Wang, J., Li, X., Xia, X. J., Zhou, Y. H., Shi, K., et al. (2014). H2O2 mediates the crosstalk of brassinosteroid and abscisic acid in tomato responses to heat and oxidative stresses. J. Exp. Bot. 65, 4371-4383. doi: 10.1093/jxb/ eru217
Conflict of Interest Statement: The authors declare that the research was conducted in the absence of any commercial or financial relationships that could be construed as a potential conflict of interest.

Copyright (c) $2016 \mathrm{Li}$, Ahammed, Li, Bao, Yu, Huang, Yin and Zhou. This is an open-access article distributed under the terms of the Creative Commons Attribution License (CC BY). The use, distribution or reproduction in other forums is permitted, provided the original author(s) or licensor are credited and that the original publication in this journal is cited, in accordance with accepted academic practice. No use, distribution or reproduction is permitted which does not comply with these terms. 